\title{
On the nature of attractive dislocation crossed states
}

\author{
R. Madec, B. Devincre, L.P. Kubin * \\ LEM, CNRS-ONERA (OM), 29 Av. de la Division Leclerc, BP 72, 92322 Chatillon Cedex, France
}

Accepted 1 June 2001

\begin{abstract}
Attractive non-coplanar dislocations that cannot react to form junctions can, nevertheless, form crossed states, i.e., junctions of null length. Such configurations have recently been described by Wickham and co-workers as an output of numerical simulations. The physical origin of the crossed states is cleared out and their conditions of occurrence are calculated within a simplified elastic frame. The results are further discussed by comparison with mesoscopic simulations of intersecting dislocations in fcc and bcc crystals. (c) 2002 Elsevier Science B.V. All rights reserved.
\end{abstract}

\section{Introduction}

The intersections of attractive, non-coplanar dislocations are the main cause of strain hardening in crystals [1]. The reactions of attractive dislocations and the properties of the resulting dislocation junctions have been in the past the object of extensive investigations, performed within the frame of linear, isotropic, elasticity theory. However, the calculation of the shapes of flexible interacting dislocations is extremely complex [2]. The available studies are thus based on rather strong approximations, but nevertheless lead to reasonable estimates regarding the stress needed to destroy

\footnotetext{
${ }^{*}$ Corresponding author. Tel.: +33-1-4673-4441; fax: +33-14673-4155.

E-mail address: ladislas.kubin@onera.fr (L.P. Kubin).
}

stable junction configurations $[3,4]$. In the recent years, dislocation intersections have been examined using dislocation dynamics (DD) simulations able to treat either junction formation and destruction and the detailed equilibrium shapes of junction configurations under stress [5-7].

Recently, Wickham et al. [7,8] have reported a new configuration of attractive dislocations, which was observed during a simulation study of the short-range elastic interactions of non-coplanar dislocations. Instead of reacting to produce a junction, two attractive dislocations may remain pinned at their intersection point, forming a stable configuration called a crossed state. The domain of occurrence of these particular configurations has been investigated numerically and illustrated by examples on bcc crystals. In the present work, a physical explanation is provided for the occurrence of crossed states, which is further justified by a 
simple elastic calculation. The calculated results are compared with results obtained in fcc crystals using a new type of DD simulation and with the simulated results $[7,8]$. A short discussion is presented in Section 4.

\section{Domain of occurrence of crossed states}

\subsection{Physical origin}

Fig. 1 depicts the successive states of the formation of a junction between two dislocation segments $\mathrm{AB}$ and $\mathrm{CD}$ gliding in different slip planes. A simple geometrical argument given by Friedel [1] indicates, however, that the configuration illustrated by Fig. 1(d) is not always that of minimum energy. During the process of junction zipping, the angle between the two intersecting segments increases from its initial value $\alpha_{0}$ at the quadruple node $\mathrm{O}$ to an equilibrium value $\alpha_{j}$. The latter is governed by the condition of equilibrium of the line tensions at the triple nodes $\mathrm{M}$ and $\mathrm{N}$. The geometry imposes, however, the condition $\alpha_{j}>\alpha_{0}$. When the initial configuration is such that this condition is not realised, junction zipping cannot occur and the quadruple node $\mathrm{O}$ represents a stable configuration, i.e., a crossed state.

Yet, the real configuration of a crossed state is more complex than the one shown dotted in Fig. 1(d). Before the two interacting segments intersect each other, they experience both an attractive force along their common perpendicular direction and torque forces in their slip planes. The latter tend to locally align the segments along the direction of the incipient junction. At each step of the process, the actual shape of the segments is locally governed by the sum of the torque forces and of the resistive line tension forces that oppose any increase in line energy. Thus, to obtain the shape depicted in Fig. 1(c), the resolved interaction forces between the two segments must be sufficiently large compared to the line tension in order to allow the local bendings to occur. When this is not the case, i.e., for weak attractive interactions, the shape of the two intersecting segments is similar to the one depicted in Fig. 1(b). Junction zipping cannot occur and one obtains an attractive bound crossed state.

\subsection{Simplified elastic model}

Real junction configurations depend upon many geometrical parameters, viz the nature of the two slip systems (slip planes and Burgers vectors), the initial direction of the lines, the length of the segments and the position of their intersection point. Only simulation methods are able to average out all the possible configurations met in a strained crystal, taking into account in each case

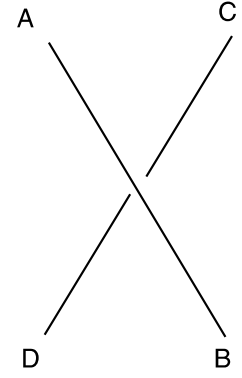

(a)

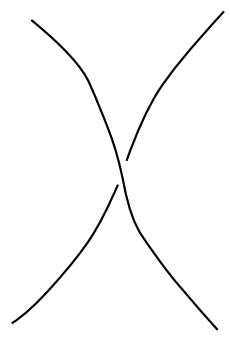

(b)



(c)

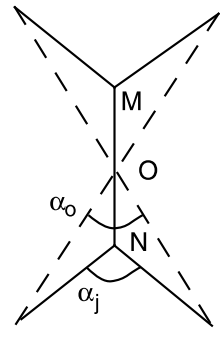

(d)

Fig. 1. Schematic view of the formation of a junction between two attractive, non-coplanar dislocation segments of finite length, AB and CD (a). At short approach distances the two segments locally bend and twist in their slip plane under the effect of their mutual attraction, thus forming an incipient junction $(\mathrm{b}, \mathrm{c})$. The junction segment is formed by a zipping process and reaches an equilibrium shape of minimum energy (d). In certain conditions, the configuration of minimum energy is a bound crossed state, similar to configuration (b). 
the complex shapes that result from the local balance of forces. The simplified models that exist in the literature [3,4] nevertheless yield a useful physical insight. They are based on several traditional assumptions: (i) As was recently confirmed by a comparison of atomistic and elastic calculations $[6,8]$, it is possible to perform realistic calculations within the frame of linear elasticity, neglecting the core energies of the dislocation lines and their modifications during the intersection process. (ii) Simplified dislocation shapes can be considered and, in what follows, the dislocation segments will be described as rigid lines. This is equivalent to neglecting the influence of the interaction forces on the segment shapes and on the logarithmic term appearing in the line energy. In such conditions, calculations can be carried out for any geometrical configuration in a straightforward manner [8]. By lack of space, we only consider here the simple case of two segments of same length that cross each other in their mid-point. As in [7], the emphasis is on the angular dependencies that have not been thoroughly investigated in previous studies.

Within the present approximation of rigid dislocations, an attractive crossed state is formed when the quadruple node is a stable equilibrium state. The two segments have initial lengths $2 \ell_{i}(i=1,2)$ and their angles with respect to the junction direction are denoted $\phi_{i}$ (cf. Fig. 2, where one portion of the total configuration is represented). Within the frame of isotropic, linear elasticity, the orientation-dependent line energies $E_{k}$ of the dislocation segments $(k=1,2)$ and of the junction $(k=j)$ are of the form

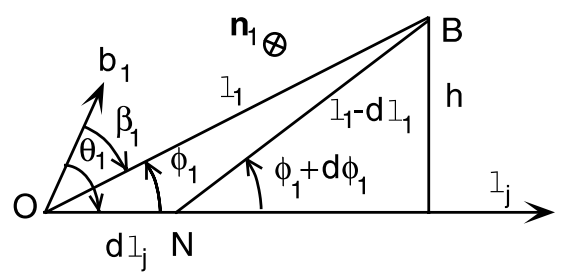

Fig. 2. A fraction of the configuration shown in Fig. 1(d), showing the zipping of a small junction segment $\mathrm{d} \ell_{j}$ from a segment of Burgers vector $b_{1}$ and initial half length $\ell_{1}$. The angular parameters are oriented as indicated with respect to the normal to the slip plane of the considered segment, $\mathbf{n}_{1}=\mathbf{b}_{1} \times \ell_{j}$.

$$
(1-v) 4 \pi E / \mu b^{2}=\ell_{i} f\left(\beta_{i}\right) ; \quad f\left(\beta_{i}\right)=1-v \cos ^{2} \beta_{i},
$$

where $\mu$ is the shear modulus, $v$ is the Poisson's ratio and the angles $\beta$ define the characters of the different segments. Another angular coordinate, $\theta$, is defined in Fig. 2(a), such that $\beta_{i}=\theta_{i}+\phi_{i}$. The moduli of the Burgers vectors of the two segments are $b_{1}=b_{2}=b$ and that of the junction, $b_{j}=n b$, depends on the type of reaction considered via the coefficient $n$. In fcc crystals, $1 / 2\langle 110\rangle$ dislocations react to form junctions of same Burgers vector and $n=1$. In bcc crystals, the reaction of two $1 / 2\langle 111\rangle$ dislocations usually produces a junction of Burgers vector $a\langle 100\rangle$ and $n=2 / \sqrt{3}$.

We consider the variation in line energy produced by the formation of a small junction segment of length $\mathrm{d} \ell_{j}$. The length of the two parent segments decreases but their character also changes. The change in total line energy is thus written as

$$
\begin{aligned}
(1-v) 4 \pi \mathrm{d} E / \mu b^{2}= & -n^{2} f\left(\beta_{j}\right) \mathrm{d} \ell_{j}+\sum_{i=1,2}\left[\mathrm{~d} \ell_{i} f\left(\beta_{i}\right)\right. \\
& \left.+\ell_{i}\left(\mathrm{~d} f / \mathrm{d} \beta_{i}\right) \mathrm{d} \beta_{i}\right] .
\end{aligned}
$$

From Fig. 2, we notice that $\ell_{i} \sin \phi_{i}=h=$ const., hence $\mathrm{d} \phi_{i}=-\left(\mathrm{d} \ell_{i} / \ell_{i}\right) \operatorname{tg} \phi_{i}$. In addition, we have $\mathrm{d} \ell_{j}=\mathrm{d} \ell_{1} / \cos \phi_{1}=\mathrm{d} \ell_{2} / \cos \phi_{2}$. Finally, we have by definition $\beta_{i}=\theta_{i}+\phi_{i}$, where the angles $\theta$ are geometrical constants. Then, $\mathrm{d} \phi_{i}=\mathrm{d} \beta_{i}$. Inserting these results into Eq. (2) and after rearrangement, we obtain:

$$
\begin{aligned}
(1-v) 4 \pi \mathrm{d} E / \mu b^{2} \mathrm{~d} \ell_{j}= & -n^{2} f\left(\beta_{j}\right) \\
& +\sum_{i=1,2}\left[f\left(\theta_{i}+\phi_{i}\right) \cos \phi_{i}\right. \\
& \left.-f^{\prime}\left(\theta_{i}+\phi_{i}\right) \sin \phi_{i}\right],
\end{aligned}
$$

where $f^{\prime}$ stands for $\mathrm{d} f / \mathrm{d} \beta$, the function $f$ being given by Eq. (1). When $\mathrm{d} E<0$, junction zipping occurs until the angles $\phi$ reach a value such that the energy is minimum. The properties of junctions, their length and the stresses necessary to unzip them can be deduced easily from such considerations. These results will be the object of a separate publication. 

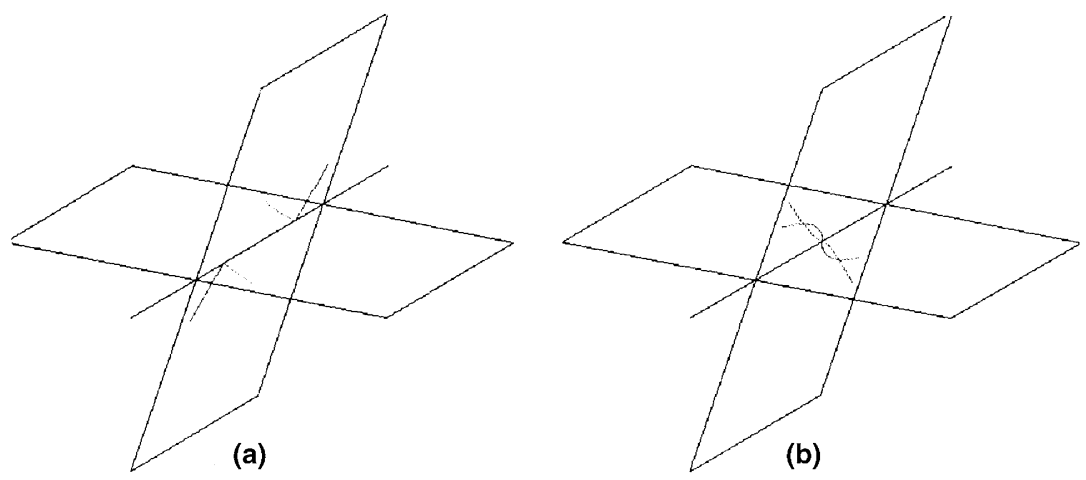

Fig. 3. Attractive intersection of two initially straight dislocation segments of length $10 \mu \mathrm{m}$, pinned at their extremities. The two interacting slip systems are (1): $1 / 2[\overline{1} 01](1 \overline{1} 1)$ and (2): $1 / 2[1 \overline{1} 0](11 \overline{1})$. (a) A junction of edge character with Burgers vector $b_{j}=1 / 2\left[\begin{array}{lll}0 & 1 & 1\end{array}\right]$ and line direction $[0 \overline{1} \overline{1}]$, formed with $\phi_{1}=153^{\circ}, \phi_{2}=45^{\circ}$. (b) A crossed state obtained with $\phi_{1}=\phi_{2}=27^{\circ}$.

In the parameter space $\left(\phi_{1}, \phi_{2}\right)$, a plot of the condition $\mathrm{d} E / \mathrm{d} \ell_{j}=0$ yields critical lobes enclosing the domain where junctions are formed (cf. Fig. 3, below). One can check easily that for fcc crystals $(n=1)$ and neglecting the orientation dependence of the line energy $(v=0)$, the critical lobes reduce to a circle of equation $\cos \phi_{1}+\cos \phi_{2}=1$ and its replicas translated by multiples of $\pm \pi$ along the two axes. Then, in such a diagram, the domain of the crossed states is necessarily situated outside the critical lobes.

An additional condition is that the two straight segments must be attractive. The corresponding critical condition can be expressed by $\mathbf{F}_{12}=0$, where $\mathbf{F}_{12}$ is the total interaction force from dislocation (1) to dislocation (2). For infinite segments, this force has been calculated by Kroupa [9]. It has the property of being constant, irrespective of the separation between the two dislocations. Thus, this expression provides a good approximation for approach distances smaller than the segment lengths. The total force is parallel to the unit vector $\mathbf{R}_{12}^{\mathrm{u}}=\mathbf{R}_{12} / \mathbf{R}_{12}$, where $\mathbf{R}_{12}$ is the shortest vector from dislocation (1) to dislocation (2). Its modulus is given by [2]

$$
\begin{aligned}
F_{12}=\propto & \left\{\frac{1}{2}\left(b_{1} \cdot \xi_{1}\right)\left(b_{2} \cdot \xi_{2}\right)-\left(b_{1} \times b_{2}\right) \cdot\left(\xi_{1} \times \xi_{2}\right)\right. \\
& \left.+\frac{1}{1-v}\left[\left(b_{1} \times \xi_{1}\right) \cdot \mathbf{R}_{12}^{\mathrm{u}}\right]\left[\left(b_{2} \times \xi_{2}\right) \cdot \mathbf{R}_{12}^{\mathrm{u}}\right]\right\},
\end{aligned}
$$

where $\xi_{1}$ and $\xi_{2}$ are the unit directions along the dislocation lines. A lengthy calculation, which is not reproduced here, allows expressing Eq. (4) in terms of the same variables as Eq. (3). The simultaneous fulfillment of the two conditions $F_{12}>0$ and $\mathrm{d} E / \mathrm{d} \ell_{j}>0$ (cf. Eq. (3)) defines the domain of occurrence of crossed states. Numerical applications are presented in Section 3 and compared with simulation results.

\section{Simulation of crossed states}

In DD simulations, and in contrast with the strong approximations made in the previous section, dislocation interactions and curvatures are taken into account. We present here results obtained with the "pure-mixed" (PM) model [10], which is an improved version of a previously developed DD simulation, the "edge-screw" model $[11,12]$. These simulations involve a discretisation of the dislocation characters on an underlying lattice, making it possible to treat large-scale configurations in single crystals. In the PM model, two mixed characters at $\pm 60^{\circ}$ are incorporated into the basic set of discretised line vectors, in addition to the screw and edge characters. This allows to treat junctions with a minimum number of rules, since all the junction directions belong to the basic set of directions. Fig. 3 shows one crossed state and one junction obtained in an fcc crystal with the help of the PM model. 
In fcc crystals, simple symmetry considerations based on the properties of the Thompson tetrahedron show that two interacting slip systems can only form two generic types of junctions. In the "mixed" configuration, one of the Burgers vectors is glissile in the slip plane of the other system and is parallel to the junction line direction. The junction is of mixed character. In the "edge" configuration, which is the one represented in Fig. 3, the two interacting Burgers vectors have no common slip plane and the junction is of edge character.

Fig. 4(a) shows the results of a set of simulations on the edge configuration of Fig. 3, in the form of a diagram in the $\left(\phi_{1}, \phi_{2}\right)$ space. The occurrence of junctions, crossed states and repulsive intersections as a function of the two angular coordinates are represented by different symbols. Superimposed to these results are shown the predictions of the approximate elastic calculations presented in Section 2. In spite of the rather strong approximations, the lobes representing the domain of junction formation are well reproduced by the condition $\mathrm{d} E / \mathrm{d} \ell_{j}=0$ (Eq. (3)). The condition of null interaction force $F_{12}=0$ (Eq. (4)) leads to a reasonable prediction for the domain of occurrence of the crossed states.

In bcc crystals, the consideration of all the possible couples of interacting slip systems leads to three types of generic configurations, two involving junctions of mixed character and one involving a junction of edge character. For instance, the interaction of the two slip systems $1 / 2\left[\begin{array}{ll}1 & 11\end{array}\right](\overline{1} 01)$ and $1 / 2[1 \overline{1} \overline{1}](0 \overline{1} 1)$ leads to the formation of a

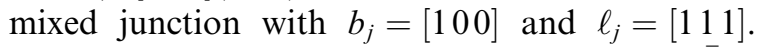
The whole configuration is glissile in the $\left(\begin{array}{lll}0 & 1 & 1\end{array}\right)$ plane. The interaction of the two slip systems $1 / 2[111](\overline{1} 01)$ and $1 / 2\left[\begin{array}{ll}1 & \overline{1}\end{array}\right](110)$ leads to the formation of a mixed sessile junction with $b_{j}=$ $[100]$ and $\ell_{j}=\left[\begin{array}{lll}1 & 1 & 1\end{array}\right]$. These two cases were examined by Wickham et al. [7]. The third case is illustrated by the interaction of the two slip sys-

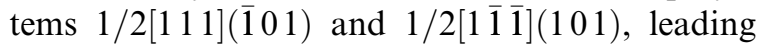
to the formation of a sessile edge junction with $b_{j}=\left[\begin{array}{lll}1 & 0 & 0\end{array}\right]$ and $\ell_{j}=\left[\begin{array}{lll}0 & 1 & 0\end{array}\right]$. The simulation of dislocation behaviour in bec metals at low temperature requires accounting for the lack of flexibility and low mobility of the screw dislocations, as a result of their particular core structure [13]. For

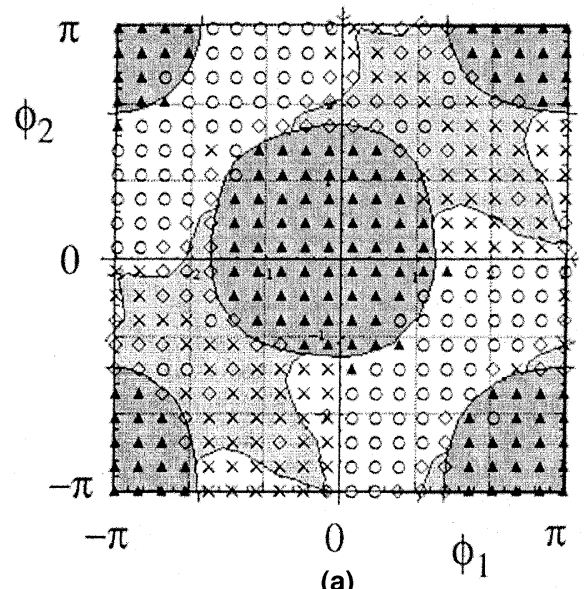

(a)

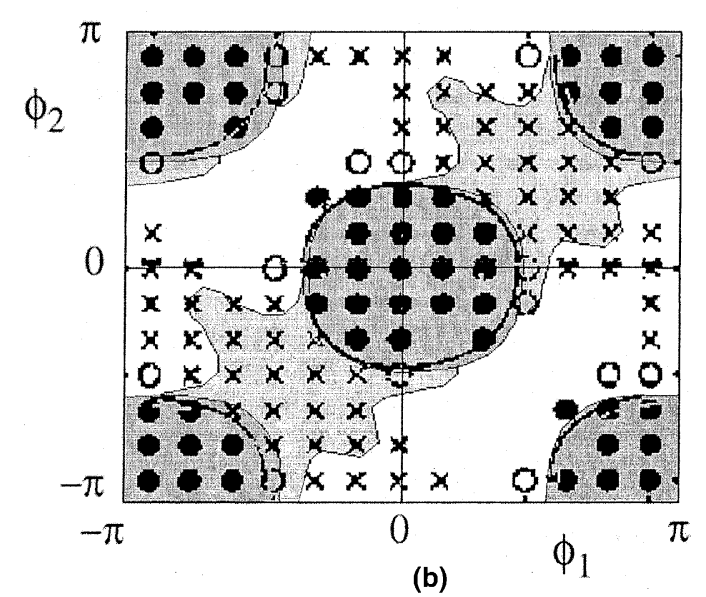

(b)

Fig. 4. Different types of short-range dislocation interactions as a function of the two angles $\phi_{1}$ and $\phi_{2}$ between the interacting dislocation lines and the junction direction. The dark and light grey areas correspond to the calculated domains of junctions and crossed states, respectively. The white areas correspond to repulsive states. The symbols show simulated results. (a) Edge configuration in an fcc crystal with same slip systems as in Fig. 3. Filled triangles: junctions, crosses: crossed states, empty circles: repulsive states, lozenges: borderline states. (b) Mixed glissile configuration in a bcc crystal. The numerical results are reproduced from Wickham et al. [7]. Filled circles: junctions, crosses: crossed states, empty spaces: repulsive interactions, empty circles: borderline cases. The calculated domain of junctions (thin lines) is practically the same as the one obtained in [8], with the help of the same elastic arguments (thick lines). 
this reason, Fig. 4(b) only shows a comparison between the present elastic model and the results of Wickham et al. [7], the core properties of the screw dislocations being ignored in both cases. As noted in [8], the domain of junction formation perfectly corresponds to the prediction of simplified elastic models. In contrast, the border between attractive and repulsive states is only qualitatively reproduced.

\section{Concluding remarks}

A physical interpretation has been given for the occurrence of attractive crossed states. These configurations are obtained when the attraction between two dislocation segments is not sufficient to overcome the resistance due to line tension effects, thus impeding the recombination of the two lines. The simplified elastic model traditionally used for treating attractive interactions between dislocations is outlined, leading to a quite reasonable comparison with the more accurate simulation results obtained using the PM model. By lack of space, the effect of an applied stress on the attractive configurations was not considered in the present study and is postponed for a further publication. Nevertheless, it is worth mentioning that a distinction can be made between "strong" junction configurations that are obtained for small values of the angles $\phi_{1}$ and $\phi_{2}$ (cf. Fig. 4), and "weak" junctions, crossed states and repulsive configurations that constitute more modest obstacles.

Although the outcome of the interaction of two arbitrary dislocations lines can be predicted, it is not desirable, to introduce this prediction in DD simulations in the form of local rules. Indeed, quite a few geometrical parameters influence the prop- erties of dislocation intersections and their combinations lead to an enormous number of possible local configurations. An objective of DD simulations should, in contrast, to let dislocation segments evolve naturally in response to their mutual interactions as much as possible. The PM model, of which some results were presented here, has been designed for this purpose with the further objective of investigating the average hardening resulting from many different types of local intersections.

\section{References}

[1] J. Friedel, Dislocations, Pergamon Press, Oxford, 1964.

[2] J.P. Hirth, J. Lothe, Theory of Crystal Dislocations, Krieger, Malabar, Florida, 1992.

[3] G. Saada, Acta Metall. 8 (1960) 841.

[4] G. Schoeck, R. Frydman, Phys. Status Solidi B 53 (1972) 661.

[5] B. Devincre, L.P. Kubin, Model. Simul. Mater. Sci. Eng. 2 (1994) 559.

[6] V.B. Shenoy, R.V. Kukta, R. Phillips, Phys. Rev. Lett. 84 (2000) 1491-1494.

[7] L.K. Wickham, K. Schwarz, J.S. Stölken, Phys. Rev. Lett. 83 (1999) 4574.

[8] L.K. Wickham, K. Schwarz, Stölken, in: I.M. Robertson, D.H. Lassila, B. Devicnre, R. Phillips (Eds.), Mat. Res. Soc. Symp. Proc., vol. 578, MRS, Warrendale, PA, 2000, p. 125.

[9] F. Kroupa, Czech J. Phys. B 11 (1961) 847.

[10] B. Devincre, L.P. Kubin, C. Lemarchand, R. Madec, in: Proceedings Dislocation-2000, Mat. Sci. Eng. A 309-310 (2001) 211.

[11] L.P. Kubin, G. Canova, M. Condat, B. Devincre, V. Pontikis, Y. Bréchet, Solid State Phenom. 23-24 (1992) 455.

[12] B. Devincre, in: H.O. Kirchner, L.P. Kubin, V. Pontikis (Eds.), Computer Simulation in Materials Science, NATO ASI E 318, Kluwer Academic Publishers, Dordrecht, 1995, p. 309.

[13] M. Tang, B. Devincre, L.P. Kubin, Model. Simul. Mater. Sci. Eng. 7 (1999) 893. 